Trinity University

Digital Commons @ Trinity

Engineering Faculty Research

Engineering Science Department

2014

\title{
A Flexible Broadband Antenna and Transmission Line Network for a Wearable Microwave Breast Cancer Detection System
}

Emily Porter

Gregory Walls

TrinityUniversity, gwalls@trinity.edu

Yahe Zhou

Trinity University, yzhou@trinity.edu

Milica Popović

Joshua D. Schwartz

Trinity University, jschwar1@trinity.edu

Follow this and additional works at: https://digitalcommons.trinity.edu/engine_faculty

Part of the Engineering Commons

\section{Repository Citation}

Porter, E., Walls, G., Zhou, Y., Popović, M., \& Schwartz, J. D. (2014). A flexible broadband antenna and transmission line network for a wearable microwave breast cancer detection system. Progress in Electromagnetics Research Letters, 49, 111-118.

This Article is brought to you for free and open access by the Engineering Science Department at Digital Commons @ Trinity. It has been accepted for inclusion in Engineering Faculty Research by an authorized administrator of Digital Commons @ Trinity. For more information, please contact 


\title{
A Flexible Broadband Antenna and Transmission Line Network for a Wearable Microwave Breast Cancer Detection System
}

\author{
Emily Porter ${ }^{1,}$, Gregory Walls ${ }^{2}$, Yahe Zhou ${ }^{2}$, Milica Popović ${ }^{1}$, and Joshua D. Schwartz ${ }^{2}$
}

\begin{abstract}
First, we report on the design, simulation and measurement of a $2-4 \mathrm{GHz}$ conformable antenna optimized for skin contact and implemented on a flexible printed circuit for integration into a wearable device. Second, we experimentally verify the suitability of appropriately long $(\sim 10 \mathrm{~cm})$ microstrip traces for the wearable system signal distribution network, which features varying radii of curvature. Consequently, the contribution of the here reported work is two-fold. First, the experimental results obtained both with breast phantoms and on-body measurements, demonstrate a return loss below $-10 \mathrm{~dB}$ in the desired frequency band. Phantom results also show a through-breast transmission coefficient of above $-40 \mathrm{~dB}$ at the centre frequency of $3 \mathrm{GHz}$. Second, and essential for signal integrity in our target application, the results show that the longitudinal curvature of such a microstrip does not increase transmission line losses.
\end{abstract}

\section{INTRODUCTION}

Microwave detection of early-stage tumors has been proposed as a complementary technique to the current standard of x-ray mammography [1]. Microwave methods offer potential benefits in that 1) they do not require ionizing radiation, allowing for frequently repeatable breast scans; 2) scans do not require breast compression; and 3) microwave components are generally lower in cost than x-ray components [1], reducing barriers to patient screening. Typical microwave systems operate either in the time-domain (with use of a pulse generator and an oscilloscope) or in the frequency-domain (with a network analyzer) [2-5]. For both methods, antenna arrays (multistatic and monostatic) and switching networks (to select which antennas are active at a given time) are common elements of the design. A wearable form factor is frequently not addressed by proposed solutions, most of which are 'bench-top' demonstrations.

In this work, we begin to adapt our early prototype detection system [6], which is built into a patient exam table, towards a wearable form factor by examining how key circuit components can be integrated into a flexible 'brassiere-like' circuit board. A wearable system has many potential cost and size advantages over the tabletop approach, which requires a patient to lie prone on specialized equipment. A reduced form factor would open doors to potential home-use or outpatient systems for breast health monitoring in which scan data is collected easily by the patient and sent to the health care provider for analysis.

In the proposed system, the requisite antennas would contact the skin directly, without the need of messy matching or immersion liquids that needs frequent replacing. The proposed architecture would integrate on a single circuit board several components that take up significant space in our first prototype: 16 end-launch antennas, a rigid radome to hold the antennas in place, an automated switching network, and all feed lines between the switches, antennas, and input/output.

\footnotetext{
Received 10 September 2014, Accepted 14 October 2014, Scheduled 23 October 2014

* Corresponding author: Emily Porter (emily.porter@mail.mcgill.ca).

1 Department of Electrical and Computer Engineering at McGill University, Montreal H3A 0E9, Canada. ${ }^{2}$ Engineering Science Department, Trinity University, San Antonio TX 78212, USA.
} 
In this work, we focus on key components of the proposed system development: the antennas and the network of transmission lines that must be used to route signals over a breast-sized and shaped surface. In our early system prototype [6], the sensing array used sixteen Traveling Wave Tapered and Loaded Transmission Line Antennas (TWTLTLAs, [7]). Since the antennas had an end-fire radiation pattern, they had to be kept perpendicular to the breast surface and housed in a rigid radome that allowed them to radiate optimally into the breast tissues. The result was a bulky and fragile bowl-shaped radome,with slots that held the antennas, while the breast of a patient lying prone and face-down on a table was positioned inside it. Further, data was collected from the multistatic antenna array using a $16 \times 2$ electromechanical switching network composed of four independent $8 \times 1 \mathrm{~s}$ and eighteen $2 \times 1 \mathrm{~s}$, an expensive and bulky solution.

Integrating both the switching network and the antenna array onto a wearable circuit board would represent a significant improvement in both system cost and ease of patient use by allowing them to wear the unit while seated or standing. In studying the integration of these elements, fundamental questions emerged about how the curvature and size of the board required by this application would affect both transmission line and antenna performance. The revised design required the routing of GHz-range signals on a curved board through an integrated solid-state switching network over long breast-scale $(\sim 10 \mathrm{~cm})$ distances. Despite a preponderance of available literature on flexible circuitry and antennas, the authors found no published reports that experimentally demonstrating how planar transmission line behavior might change with pronounced flexing along the long axis (in the direction of propagation), while classical theory predicts that additional radiative losses could occur (due a sufficiently high radius of curvature acting as a discontinuity). Thus, in this work, we present transmission data from $1-40 \mathrm{GHz}$ (well beyond our application space) for a microstrip implemented in a flexible transmission line for varying radii of curvature. Our choice to investigate microstrip (rather than a coplanar alternative) was motivated by our intention to use this geometry for the long $(\sim 10 \mathrm{~cm})$ distribution lines required of our anticipated system design, for which loss behavior, trace width and layout simplification were deciding factors. Coplanar feed lines are anticipated for the completed system's antennas but were not investigated for longitudinal curvature due to their very short lengths $(<1 \mathrm{~cm})$ in our design.

In contrast to the gaps in the literature on the flexing performance of transmission lines, several works have discussed flexible broadband antennas [8-11]. However, measurement results on a flexing antenna are rare $[8,9]$. Furthermore, works describing compact antenna arrays $[12,13]$, have shown how planar arrays can be applied to breast cancer detection; however, neither presents experimental evidence on physically flexed arrays.

Here we present the design and experimental testing of an elliptical patch antenna and a microstrip trace when flexed on surfaces of known curvature. Our findings suggest that there is a negligible increase in radiative losses for uniformly-applied radii of curvature in microstrip as observed from $1-40 \mathrm{GHz}$. We also confirm the operation of our antenna design with acceptable return loss for the $2-4 \mathrm{GHz}$ band, under flat and flexed conditions.

\section{EXPERIMENTAL SETUP}

Our long-term goal is to design a wearable system that contains 16 antennas conformed to a curved printed circuit board (PCB) that is approximately breast-shaped. The antennas will be evenly distributed around the board so as to maximize coverage of the breast. Transmission lines to and from all antennas will be routed on the surface of the flexible circuit board in microstrip technology and a microstrip-to-coplanar layer transition will be implemented at each antenna feed point. The microstrip distribution network and antennas will both be flexed to conform to the breast.

The design target for this wearable system is a breast monitoring application: healthy patients will undergo regular, frequent breast scans, enabling future scans to be compared to earlier ones to identify if any tissue abnormalities are developing [6]. In this way, the system will be calibrated with the initial breast scan of a given patient, and the data set will consist of a later scan of that same patient. The following paragraphs describe the microstrip transmission line networks, which will be tested in conformed implementations, followed by a discussion of the antennas.

For our transmission-line testing, we wished to investigate any potential losses that could be incurred by a high-degree of longitudinal flexing, so we constructed an 8.9-cm long $50 \Omega$ microstrip 
trace of width $0.28 \mathrm{~mm}$ on a flexible Rogers Ultralam $\AA$ ) 3850 board (relative permittivity $\varepsilon_{r}=2.9$ at $10 \mathrm{GHz}$ ) of $101 \mu \mathrm{m}$ thickness with $1 / 2 \mathrm{oz}$. bare copper metallization. The traces were connectorized using end-launch SMA adaptors and connected to a calibrated 40-GHz Anritsu 37369A vector network analyzer (VNA). $S$-parameter measurements were recorded for various radii of curvature by placing and affixing the microstrip to the curved surface of several 3-D printed cylindrical structures (see Fig. 1) of differing radii of curvature of interest, ranging from $60 \mathrm{~mm}$ to $20 \mathrm{~mm}$. A baseline $S$-parameter measurement for zero curvature was obtained for comparison.

We then designed a flexible, compact antenna based on an elliptical monopole patch with a coplanar waveguide feed, similar to the one presented in [11], except designed for the 2-4 GHz range and for use in close contact with skin. As was shown in [14], the $2-4 \mathrm{GHz}$ range is good trade-off between the high resolution yet high attenuation at higher frequencies and the undesirable multiple reflections caused by low attenuation (high penetration) at lower frequencies. The proposed design improves over typical antennas used for this application, for example in [4,7], and [15-17], by being simultaneously compact, simple and low-cost to fabricate, extremely flexible, and exhibiting wideband broadside radiation. This antenna type was also chosen for its omnidirectional radiation pattern, as, especially with a limited number of sensors, it is important to have the radiation beams of the transmitter and receiver overlap at all locations in the breast for several possible transmitter and receiver combinations, otherwise a potential tumor could be overlooked [18].

As in the case of the printed microstrip traces, the antenna was designed on $101 \mu \mathrm{m}$ Ultralam 3850 to exhibit an input impedance of $50 \Omega$ (matched to the chosen feed line and connector impedances) when placed directly against the skin. In numerical simulation, the antenna was modeled as flat and was covered on one side with layers mimicking typical properties of breast tissues: $2.5 \mathrm{~mm}$ of skin $\left(\varepsilon_{r}=40\right.$, conductivity $\sigma=2 \mathrm{~S} / \mathrm{m}$, and loss tangent $\tan \delta=0.2996)$, and $80 \mathrm{~mm}$ of high-adipose content tissue $\left(\varepsilon_{r}=10, \sigma=0.4 \mathrm{~S} / \mathrm{m}, \tan \delta=0.2397\right)$. These dielectric properties were chosen to match reported tissue properties at the centre frequency of $3 \mathrm{GHz}$; the ranges of measured dielectric properties for breast tissues can be found in [19] (for skin), and in [20] (for low- and high-adipose content tissues). We performed simulation and measurement of the antenna behavior on a flat phantom surface to confirm its behavior before proceeding to measurements under various curvatures.

Numerical optimization for lowest return loss suggested a coplanar waveguide (CPW) feed having a width of $1.05 \mathrm{~mm}$ and a slot width of $0.111 \mathrm{~mm}$ on either side. The elliptical patch had a major radius of $9.5 \mathrm{~mm}$ and an ellipse ratio of 1.4 . The antenna footprint was $24 \times 32 \mathrm{~mm}^{2}$, not including the feeding section used to connectorize to a right-angle coaxial cable for SMA-connectivity and ease of network analysis. In its intended application, an array of these antennas will be implemented on a multilayer flexible circuit board, with the signals to and from the antennas and feed lines routed ultimately to a connectorized board edge on the top layer in microstrip. As the curvature of each individual antenna in the array will be unique, we optimized under flat conditions and then evaluated the impact of various

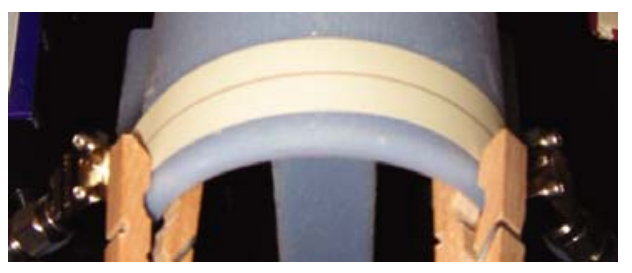

Figure 1. Photograph of the fabricated microstrip trace curved over a surface.

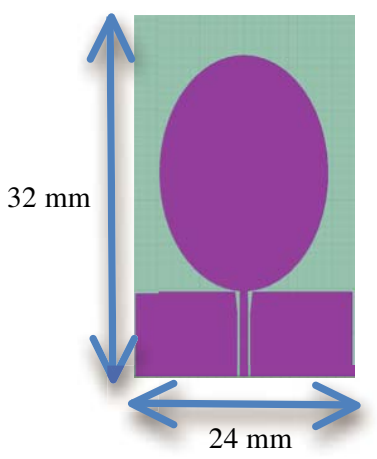

(a)

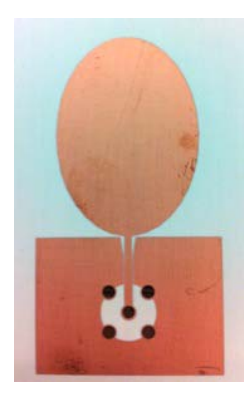

(b)
Figure 2. (a) Numerical simulation model of the antenna and (b) a photograph of the fabricated antenna with a vertical coaxial feed. The antenna measures $24 \times 32 \mathrm{~mm}^{2}$. 
curvatures. From simulations, we are able to determine that the antenna, when in contact with skin, has a near-field radiation pattern that is near omnidirectional and broadside; the precise radiation pattern will vary depending on the complex geometry of the breast with which the antenna is contacting. We also note that the radiation efficiency is $47 \%$.

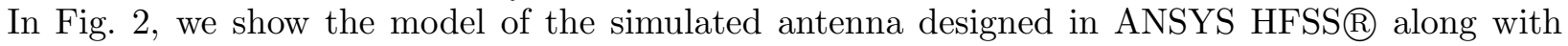
a photograph of the corresponding fabricated antenna, which includes via holes for a 5-pin coaxial connection. In measurements, synthetic breast tissue-mimicking materials ("phantoms") were needed in order to test the antenna. All phantoms used in this work are created using the methods and materials demonstrated in [21].

\section{RESULTS}

In Fig. 3 we present experimentally measured results for an $8.9 \mathrm{~cm}$ microstrip trace with a varying radius of longitudinal curvature. The topmost graph presents the transmission losses $\left(S_{12}\right)$ for various radii and the flat reference, while the bottommost graph plots the differences in measurement for these radii and the reference. Our results serve to confirm that the transmission behaviors of the traces are nearly identical (less than $1 \%$, or $<0.1 \mathrm{~dB}$ difference) for any radius of curvature across the investigated bandwidth of 1-40 GHz. No discernable correlation was noted between the $S_{12}$ results and the radii of curvature, indicating that physical factors of simply moving the microstrip from one curved surface to the next were more disruptive than the increased curvature itself. The slight ripple evident in the response has a ripple frequency of approximately $1.1 \mathrm{GHz}$, and can be directly attributed to harmonics of a long-path resonance along the entire microstrip due to the slight mismatch introduced at the endlaunch connectors. This can be verified using the classical formula (1) assuming the resonance occurs when the path length $L=8.9 \mathrm{~cm}$ is one-half of a wavelength.

$$
f=\frac{c}{2 L \sqrt{\varepsilon_{e f f}}}
$$

In this equation, $c$ is the speed of light in a vacuum and $\varepsilon_{\text {eff }}$ the effective relative permittivity of the quasi-TEM mode of the microstrip. In this case, $\varepsilon_{\text {eff }}$ is about 2.35 based on the properties of the substrate.

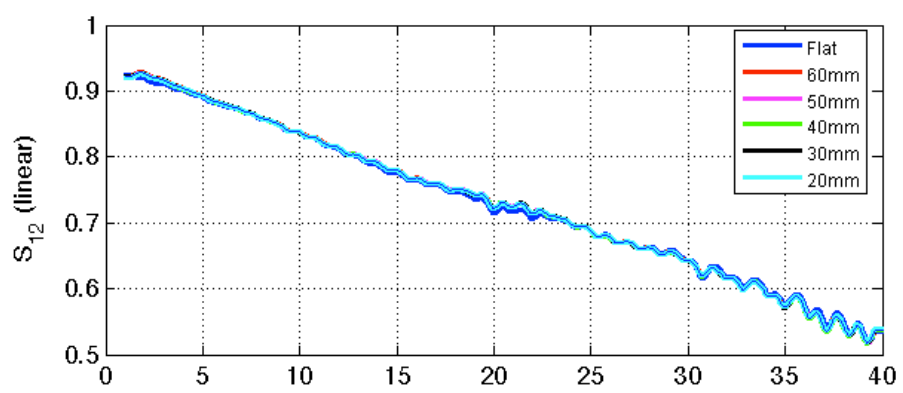

(a)

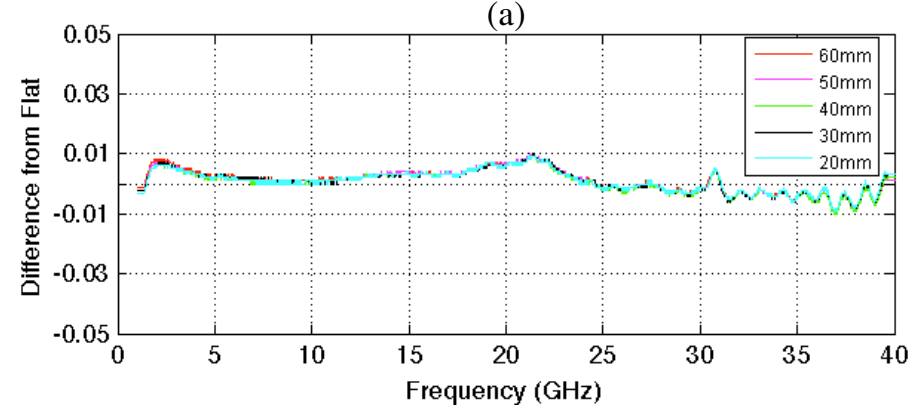

(b)

Figure 3. (a) Measured transmission behavior of an 8.9-cm flexible microstrip experiencing various radii of longitudinal curvature, and (b) the difference from a flat reference measurement. 
Even in the most severe case of bending and at the highest recorded frequency, there was no apparent correlation between the bend radius and the transmission, with a negligible difference noted from a flat reference measurement and no noteworthy distinction between the various measurements. The authors obtained a similar result for a grounded CPW trace with the same center trace width and length. This result is not unexpected and serves to demonstrate that the influence of longitudinal curvature on microstrip operation is negligible.

We next present the flexed antenna measurement data. The first set of measurements were performed on cylindrical, tissue-mimicking phantoms (without skin) meant to allow us to isolate flexing on different axes. The phantoms have a height of $8.23 \mathrm{~cm}$, and radii $r 1=8.23 \mathrm{~cm}, r 2=12 \mathrm{~cm}$, and $r 3=16.5 \mathrm{~cm}$. These sizes are chosen to approximate typical breast curvatures. The return loss $\left(S_{11}\right)$ was measured for two orientations of the antenna with respect to a cylinder: orientation 01 denotes that the antenna's main axis (long axis of the elliptical patch) is aligned with the axis of the cylinder; orientation 02 is a $90^{\circ}$ rotation with respect to 01 wherein the antenna axis is perpendicular to the cylinder axis as shown in Fig. 4.
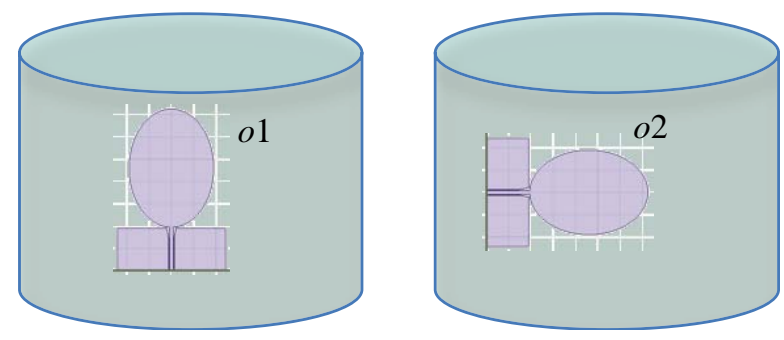

Figure 4. Diagram of the antenna orientations for $S_{11}$ measurements on cylindrical phantoms.

Figure 5 illustrates the simulated and measured $S_{11}$ for the flexible antenna over the $1.5-4.5 \mathrm{GHz}$ range. The measured data demonstrates that the return loss is well below $-10 \mathrm{~dB}$ over the entire $2-$ $4 \mathrm{GHz}$ range of interest for all measured antenna positions and curvatures. The $S_{11}$ did not deteriorate significantly with higher levels of flexing in either position but did experience up to $10 \mathrm{~dB}$ of change (for example, see the traces for $r 3$ at $2.7 \mathrm{GHz}$ ).

In Fig. 6, we depict the measurement scenario for antennas curved against the side of a hemispherical $(r=6.5 \mathrm{~cm})$ tissue-mimicking phantom. This breast phantom has curvature in both dimensions, and also features a skin-mimicking layer. Both the skin and interior breast tissues are designed to have dielectric properties that approximate the actual tissue properties. Fig. 7 plots the measured $S_{11}$ for an antenna conformed to the hemispherical phantom. The measurement results confirmed that the return loss was sufficiently below $-10 \mathrm{~dB}$ for proper antenna operation in the $2-4 \mathrm{GHz}$ target range, for both the curved surface and the flat reference. The values were in the same range as those of Fig. 5 for the cylindrical (one-dimensional bending), suggesting that the amount of curving needed for the antenna to flex around the phantom is not detrimental to the antenna's radiative behavior.

We also validated the transmission capabilities of the proposed antenna array through the breast tissues by measuring the transmission coefficient $S_{21}$ between two of the flexible antennas located on opposite sides of the hemispherical phantom, at three distances of approximately 9,10 , and $11 \mathrm{~cm}$ apart, as shown in Fig. 6.

These distances are representative of those that could be expected for antennas co-located in a coronal plane around a breast with a $\mathrm{D}$ cup size. In this measurement, the antennas were oriented similarly: curved around the phantom with the ellipse axis parallel to the plane of the chest wall. As the $S_{21}$ would understandably vary for different relative positions of the transmit and receive antennas within the array, the parameter is measured here with antennas directly across each other on the hemisphere at different 'heights', where the flat chest wall is taken to be zero height. Further, antennas that are closer together are, by the geometry of the curved surface to which they are conformed, necessarily at more of an angle relative to each other than antennas that are further apart (which are almost parallel). Thus, the polarization for these transmit-receive antenna pairs does not match up as well, which can result in a minor loss in the amplitude of the transmitted signal. However, due to the complex scenario, with multiple interfaces at oblique angles and corresponding wave scattering, it is not straightforward to 


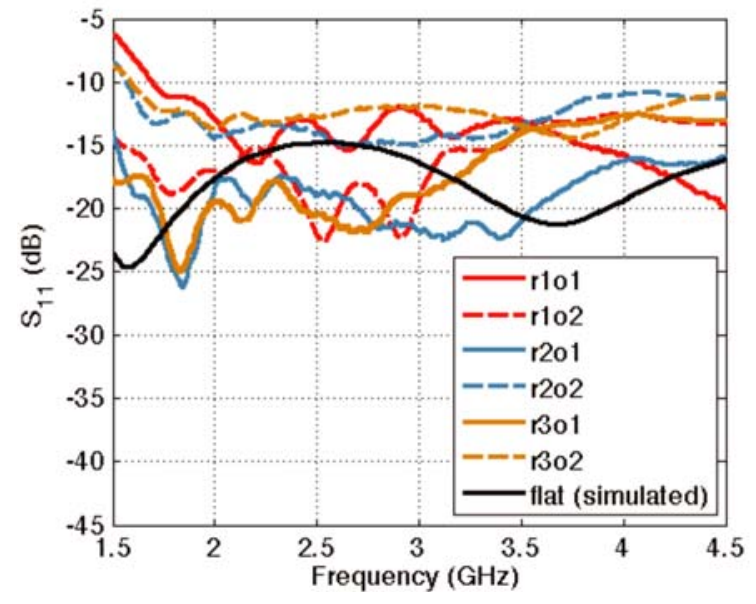

Figure 5. Simulated $S_{11}$ (black) for a flat antenna compared to the measured $S_{11}$ for an antenna curving along a phantom of varying radii of curvature, in two different dimensions: radius $r 1=8.23 \mathrm{~cm}$ (red), $r 2=12 \mathrm{~cm}$ (blue), $r 3=$ $16.5 \mathrm{~cm}$ (orange); orientations $o 1$ (solid) and $o 2$ : (dashed). Simulated and measured $S_{11}$ is below $-10 \mathrm{~dB}$ for all tested scenarios over $2-4 \mathrm{GHz}$.

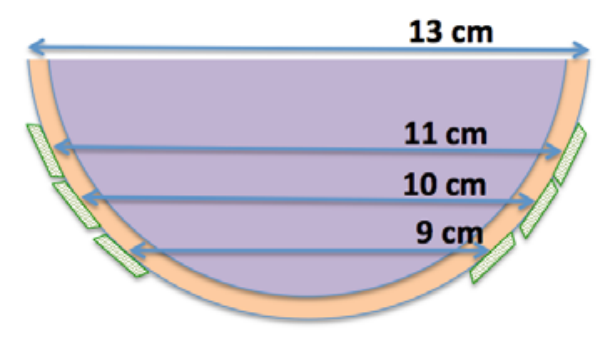

Figure 6. Sample antenna locations around a hemispherical breast phantom for $S_{11}$ and $S_{21}$ measurements. [Not to scale.] The orange layer is skin, purple is the fat-like tissue inside the phantom, and the green rectangles represent the antennas conformed to the breast surface. $S_{21}$ measurements are conducted on antennas that are located on opposite sides of the phantom: Position $1=$ antennas $11 \mathrm{~cm}$ apart; position $2=$ antennas $10 \mathrm{~cm}$ apart; position $3=$ antennas $9 \mathrm{~cm}$ apart.

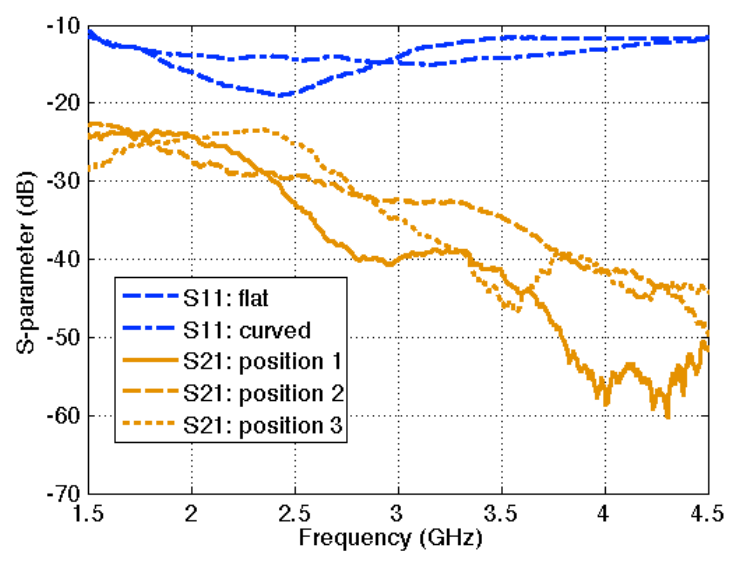

Figure 7. Measured $S_{11}$ (blue) for the proposed antenna on both flat (dash) and curved (dash-dot) tissue-like surfaces. The $S_{11}$ is below the $-10 \mathrm{~dB}$ mark for both measurements. Also plotted is the measured $S_{21}$ (orange) for three positions of antenna pairs, curved around the phantom at different locations as in Fig. 6. Position $1=$ antennas $11 \mathrm{~cm}$ apart; position $2=$ antennas $10 \mathrm{~cm}$ apart; position $3=$ antennas $9 \mathrm{~cm}$ apart.

identify if the decrease in attenuation for closer antennas is offset by the polarization mismatch between their orientations or not. The resulting data is also plotted in Fig. 7. The $S_{21}$ is strongly dependent on the relative antenna positions, as is typical in such arrays, and varies from approximately $-25 \mathrm{~dB}$ to $-28 \mathrm{~dB}$ at $2 \mathrm{GHz}$ up to -41 to $-58 \mathrm{~dB}$ at $4 \mathrm{GHz}$. As expected, the transmitted power is attenuated for higher frequencies due to increased propagation loss, which is measured to be twice as high at $4 \mathrm{GHz}$ as it is at $2 \mathrm{GHz}$ for this particular tissue-mimicking material. A similar attenuation in $S_{21}$ over the $2-4 \mathrm{GHz}$ range is shown in [5], for measurements on a 55-year old volunteer. 


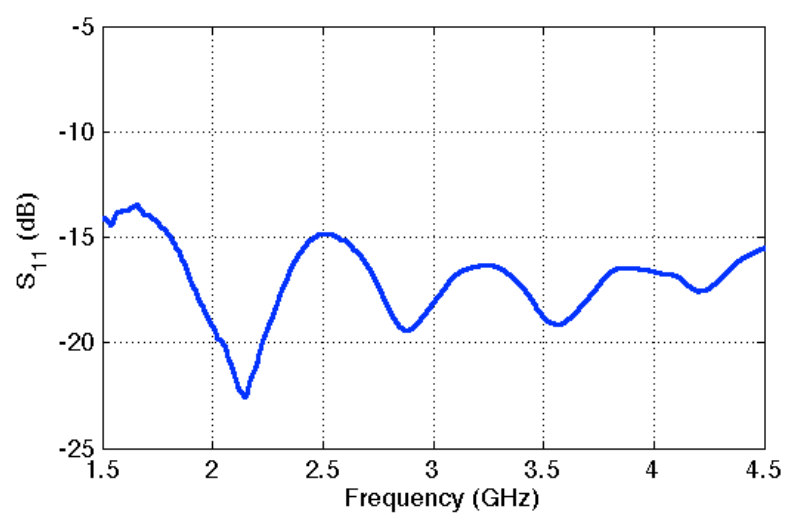

Figure 8. $S_{11}$ measurement performed on-body. The $S_{11}$ is well below the $-10 \mathrm{~dB}$ level over all frequencies of interest.

Lastly, we show the $S_{11}$ measured on-body of an actual patient volunteer. For this measurement, the antenna was pressed onto the skin in a curved position approximately similar to that of either one of the antennas of position 3 in Fig. 6. This result is provided in Fig. 8 and demonstrates that the $S_{11}$ is well below the required $-10 \mathrm{~dB}$ for the entire frequency range of interest. The measured data on-body shows that the return loss is less constant over the frequency range than it was for phantom measurements; however, the average return loss value over our frequency range is in line with that of curved measurements on the hemispherical phantom. We note that direct comparison between the phantom measurements presents a challenge as we do not know the precise dielectric properties of the skin and interior tissues for the patient volunteer, and as such they may be somewhat dissimilar to the phantom properties. Further, the exact curvature of the antenna is difficult to calculate when the antenna is located on-body because it conforms to the skin, which is an irregularly shaped object that is neither cylindrical nor spherical.

\section{CONCLUSION}

In this work we have illustrated the feasibility of using transmission lines and antennas on flexible surfaces suitable for a more integrated approach to microwave breast-cancer detection. Verifying the performance of these elements on curved breast-like surfaces and on-body moves us closer to an integrated, low-cost and patient-friendly detection system. Near-future work in integrating the system will involve a full system demonstration and miniaturization of its active components (e.g., the pulse generation and measurement apparatus).

\section{ACKNOWLEDGMENT}

This work was supported by the Natural Sciences and Engineering Research Council of Canada (NSERC), and Partenariat de recherche orientée en microélectronique, photonique et télécommunications (PROMPT).

\section{REFERENCES}

1. Nikolova, N. K., "Microwave biomedical imaging," Wiley Encyclopedia of Electrical and Electronics Engineering, 1-22, Apr. 2014.

2. Zeng, X., A. Fhager, M. Persson, P. Linner, and H. Zirath, "Accuracy evaluation of ultrawideband time domain systems for microwave imaging," IEEE Trans. Antennas Propag., Vol. 59, No. 11, 4279-4285, Nov. 2011.

3. Klemm, M., I. J. Craddock, J. A. Leendertz, A. Preece, and R. Benjamin, "Radar-based breast cancer detection using a hemispherical antenna array-experimental results," IEEE Trans. Antennas Propag., Vol. 57, No. 6, 1692-1704, 2009. 
4. Meaney, P. M., M. W. Fanning, D. Li, S. P. Poplack, and K. D. Paulsen, "A clinical prototype for active microwave imaging of the breast," IEEE Trans. Microw. Theory Techn., Vol. 48, No. 11, 1841-1853, Nov. 2000.

5. Bourqui, J., J. Garrett, and E. C. Fear, "Measurement and analysis of microwave frequency signals transmitted through the breast," International Journal of Biomedical Imaging, Vol. 2012, 1-11, Article ID 562563, 2012.

6. Porter, E., E. Kirshin, A. Santorelli, M. Coates, and M. Popović, "Time-domain multistatic radar system for microwave breast screening," IEEE Antennas Wireless Propag. Lett., Vol. 12, 229-232, 2013.

7. Kanj, H. and M. Popović, "A novel ultra-compact broadband antenna for microwave breast tumor detection," Progress In Electromagnetics Research, Vol. 86, 169-198, 2008.

8. Yoon, H. K., W. S. Kang, Y. J. Yoon, and C.-H. Lee, "A flexible UWB antenna attachable to various kinds of materials," Proc. IEEE International Conference on Ultra-Wideband (ICUWB), 204-209, Singapore, Sep. 24-26, 2007.

9. Peter, T. and R. Nilavan, "A study on the performance deterioration of flexible UWB antennas," Proc. Loughborough Antennas \& Propagation Conf., 669-672, Loughborough, UK, Nov. 16-17, 2009.

10. Karacolak, T. and E. Topsakal, "A double-sided rounded bow-tie antenna (DSRBA) for UWB communication," IEEE Antennas Wireless Propag. Lett., Vol. 5, 446-449, 2006.

11. Nikolaou, S., D. E. Anagnostou, G. E. Ponchak, M. M. Tentzeris, and J. Papapolymerou, "Compact ultra wide-band (UWB) CPW-fed elliptical monopole on liquid crystal polymer (LCP)," Proc. IEEE Antennas and Propagation Society International Symposium, 4657-4660, Jul. 9-14, 2006.

12. Sugitani, T., S. Kubota, A. Toya, X. Xiao, and T. Kikkawa, "A compact $4 \times 4$ planar UWB antenna array for 3-D breast cancer detection," IEEE Antennas Wireless Propag. Lett., Vol. 12, 733-736, 2013.

13. Bassi, M., M. Caruso, M. S. Khan, A. Bevilacqua, A.-D. Capobianco, and A. Neviani, "An integrated microwave imaging radar with planar antennas for breast cancer detection," IEEE Trans. Microw. Theory Techn., Vol. 61, No. 5, 2108-2118, May 2013.

14. Santorelli, A., M. Chudzik, E. Kirshin, E. Porter, A. Lujambio, I. Arnedo, M. Popović, and J. D. Schwartz, "Experimental demonstration of pulse shaping for time-domain microwave breast imaging," Progress In Electromagnetics Research, Vol. 133, 309-329, 2013.

15. Bourqui, J., M. Okoniewskiand, and E. C. Fear, "Balanced antipodal vivaldi antenna with dielectric director for near-field microwave imaging," IEEE Trans. Antennas Propag., Vol. 58, No. 7, 23182326, Jul. 2010.

16. Tiang, S. S., M. Sadoon, T. F. Zanoon, M. F. Ain, and M. Z. Abdullah, "Radar sensing featuring biconical antenna and enhanced delay and sum algorithm for early stage breast cancer detection," Progress In Electromagnetics Research B, Vol. 46, 299-316, 2013.

17. Moussakhani, K., R. K. Amineh, and N. K. Nikolova, "High-efficiency TEM horn antenna for ultra-wide band microwave tissue imaging," Proc. 2011 IEEE International Symp. Antennas and Propagation (AP-S), 127-130, Spokane, Washington, U.S.A., Jul. 3-8, 2011.

18. Craddock, I. J., M. Klemm, J. Leendertz, A. W. Preece, and R. Benjamin, "An improved hemispherical antenna array design for breast imaging," Proc. 2nd European Conference on Antennas and Propagation (EUCAP), 1-5, Edinburgh, Scotland, Nov. 11-16, 2007.

19. Gabriel, S., R. W. Lau, and C. Gabriel, "The dielectric properties of biological tissues III: Parametric models for the dielectric spectrum of tissues," Phys. Med. Biol., Vol. 41, No. 11, 22712293, Nov. 1996.

20. Lazebnik, M., M. McCartney, D. Popovic, C. B. Watkins, M. J. Lindstrom, J. Harter, S. Sewall, A. Magliocco, J. H. Booske, M. Okoniewski, and S. C. Hagness, "A large-scale study of the ultrawideband microwave dielectric properties of normal breast tissue obtained from reduction surgeries," Phys. Med. Biol., Vol. 52, 2637-2656, 2007.

21. Garrett, J. and E. Fear, "Stable and flexible materials to mimic the dielectric properties of human soft tissues," IEEE Antennas Wireless Propag. Lett., Vol. 13, 599-602, 2014. 